\title{
Impact of different wood chip species (oak, acacia and cherry) on evolution of individual anthocyanins, chromatic characteristics and antioxidant capacity in model wine solutions
}

\author{
António M. Jordão ${ }^{1, a}$, Viriginia Lozano², Ana C. Correia ${ }^{1}$, and María L. González-SanJosé2 \\ ${ }^{1}$ Instituto Politécnico de Viseu (CI\&DETS), Escola Superior Agrária, Estrada de Nelas, Ranhados, 3500-606 Viseu, Portugal \\ ${ }^{2}$ Universidad de Burgos, Facultad de Ciencias, Departamento de Biotecnologia e Ciencia de los Alimentos, Plaza Misael Bañuelos s/n, \\ 09001 Burgos, España
}

\begin{abstract}
The aim of present work was to investigate the impact of different oak $(Q$. alba, $Q$. robur and $Q$. pyrenaica), acacia and cherry wood chips on evolution of individual anthocyanin content, chromatic characteristics and antioxidant capacity by the use of model wine solutions. According to the results obtained, model wine solutions containing acacia and especially cherry wood extracts showed more evident changes of individual anthocyanin composition, especially due to the formation of large number of new direct condensation anthocyanins, than model wines with oak extracts. These results were accompanied also by a higher color degradation and a lower antioxidant potential of solutions containing the different wood chip extract species, compared with solutions containing only anthocyanin alone. This research contributes to a better knowledge about the color evolution of red wines when they are aging in contact with new alternative wood species, especially acacia and cherry.
\end{abstract}

\section{Introduction}

During red wine aging in contact with oak wood, phenolic compounds, mainly anthocyanins, undergo several changes that may lead to color loss or stabilization. Previous studied showed that the origin or variety of the oak together with the specific practices of each coopery, in addition to the age of the barrels and the wine aging time in contact with oak wood, play a key role in the formation of "new pigments" which could improve chromatic characteristic of wines [1-3]. Despite the advances made in the evolution of red wine color and composition during wine aging in contact with different oak wood species [4-6], there is a scare knowledge about the potential impact on anthocyanin, chromatic characteristics and antioxidant capacity evolution when red wines are aging in contact with other non-oak wood species. This is an important topic because the high demand of oak wood products has an ecological impact on harvesting of oak trees in forest where the replacement of trees is not guaranteed. Thus, the use of potential alternative woods, such as acacia and cherry, may be an interesting option for potential use in red wine aging process.

The aim of present work was to investigate the impact of oak (French, American and Iberian species), acacia and cherry wood chips on evolution of individual anthocyanin content, chromatic characteristics and antioxidant capacity by the use of model wine solutions.

\section{Material and methods}

\subsection{Wood chip samples}

The wood chip samples used were: acacia (Acacia pseudorobinia) chips purchased by SAI company (Paredes, Portugal), cherry (Prunus avium) French and American oak (Quercus petraea and Quercus alba, respectively) chips purchased by AEB Bioquímica company (Viseu, Portugal), and Iberian oak chips from Portugal (Quercus pyrenaica) purchased by J.M. Gonçalves company (Palaçoulo, Portugal), with medium toasting (20 min at $160-170^{\circ} \mathrm{C}$ ), a particle size of $8 \mathrm{~mm}$ and submitted to a natural drying.

\subsection{Model wine solutions}

Aliquots of a red model wine solution (12\% ethanol, adjusted to $\mathrm{pH} 3.5$ with tartaric-tartrate buffer and containing grape isolated anthocyanins) was added with different wood chip concentrated extracts (final concentration corresponding to $4 \mathrm{~g}$ wood chips/L wine) from oak, acacia and cherry species obtained after 15 and 30 days of extraction. After mixing, the different solutions were stored during 30 days under controlled temperature (around $14^{\circ} \mathrm{C}$ ) and darkness conditions. All assays were made in duplicate.

\subsection{Methods}

By the use of HPLC-DAD, the individual anthocyanin content evolution was analyzed followed the methodology described by Pérez and González-Sanjosé [7], while chromatic characteristics evolution (color intensity) was studied by spectrophotometric methodologies [8], and

\footnotetext{
a e-mail: antoniojordao@esav.ipv.pt
} 


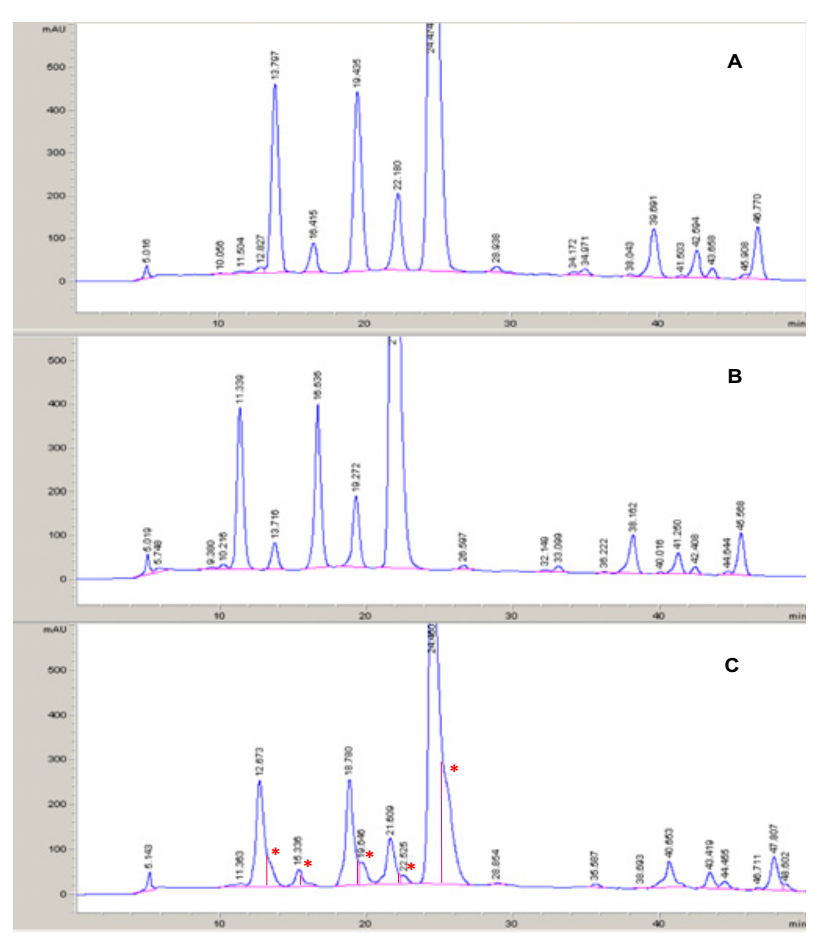

Figure 1. Changes of the individual anthocyanin profile (HPLC) after mixing a model wine solution, containing anthocyanins, with a wood chip extracts. (A) model wine solution containing anthocyanin extract alone; (B) model wine solution containing anthocyanin extract and oak wood chip extract after 15 days of storage; (C) model wine solution containing anthocyanin extract and oak wood chip extract after 30 storage days; (*) new pigments formed during storage.

by the use of CieLAB* coordinates [9]. To distinguish the color more accurately, the color difference was also calculated using the following formula: $\left(\Delta E=\left[\left(\Delta L^{*}\right)^{2}+\right.\right.$ $\left.\left.\left(\Delta a^{*}\right)^{2}+\left(\Delta b^{*}\right)^{2}\right]^{1 / 2}\right)$. Finally, the antioxidant capacity evolution was investigated by the use of ABTS and FRAP assays, applying the conditions optimized by Rivero et al. [10]. All analyses were done in triplicate.

\section{Results and discussion}

\subsection{Individual anthocyanins composition}

According to obtained results (Fig. 1), levels of newly formed anthocyanins are notable visible after 30 days of contact of model wine solutions with wood extracts.

During the storage time considered a decrease of free anthocyanins was detected in all model wine solutions (Fig. 1A, 1B and 1C). In addition, new pigments are formed in the solutions containing anthocyanin extract and wood extract (Fig. 1C). These new products formed occurred in all solutions containing anthocyanin extract and different extracts obtained from the different wood chip specie used (oak, cherry and acacia). The number of new products formed (including the anthocyanin pyruvic derivative products) and their concentration increase with the storage time in all model wine solutions containing anthocyanin extract and wood chip extracts. However, the majority of the direct condensation products formed were detected in solutions containing anthocyanin extracts and acacia and cherry wood chip extracts after 15 storage days (data not show). The results obtained in our work are in agreement with the research work previously developed by Sáchez et al. [11] where was analyzed the effect of microoxygenation and wood type on the phenolic composition and color parameters of red wines. In addition, Jordão et al. [3] also detected new HPLC peaks corresponding to new degradation products in solutions containing a mixture of malvidin-3-glucoside and ellagic acid extracted from oak wood and also in solutions containing malvidin-3glucoside and oak wood extract. These new compounds formed increased slightly during 64 storage days.

Thus, our work points out the importance of wood components not only from oak wood species, but also from acacia and cherry wood species in the transformation of anthocyanins, as well as in reactions that occur during the red wine aging in contact with the different wood species. It is also possible that the new products formed could participate in the color changes that usually occurred in red wines aged in wood barrels or in contact with wood chips.

\subsection{Chromatic parameters}

Color changes are one of the greatest modifications occurring during the wood aging of red wines. Anthocyanins are able to reacts with compounds extracted from wood but also with other compounds formed by redox potential changes, induced by wood components. Under these conditions, anthocyanins transformation may lead to color loss or stabilization, with the corresponding negative or positive effect on wine quality. This fact can be valuated through the measure of color parameters as color intensity, tonality and the CieLAB* parameters.

The color intensity evolution quantified in the model wine solutions containing the different wood chip extract species is illustrated in Fig. 2. After 30 days of storage, a general decrease of color intensity was observed in all the studied mixtures of red wine model solutions and wood extracts. This result was also obtained independently of the extraction time used for obtained the different wood extracts (15 and 30 extraction days). However, quantitative differences were detected among samples by factors, wood extraction time and wood type. Higher decreases of color intensity were always observed in mixtures containing wood extracts obtained after 30 days of chips extractions. So, mixtures with wood extracts obtained after 15 extraction days, showed significant decrease of color intensity only after 30 days of storages. The color reduction detected, may be due to the wood components that were extracted from wood chips that induce the rate of anthocyanin decline and consequently induced a color decrease (especially red color component). Previously Jordão et al. [2] reported a malvidin-3-glucoside declined more rapidly in the presence of oak wood extract than alone and consequently a more evident decrease of red color. In addition, it has been reported that numerous anthocyanin adducts are formed during red wine aging in oak wood barrels [12]. These new compounds presumably have an influence on the chromatic characteristics of red wines.

Another interesting result obtained is related with the impact of the different wood chip extract species on color intensity decrease. In fact, the color decrease was significantly more pronounced for the red model wine solutions mixed with the cherry wood extracts (particularly for the wood extract obtained after 30 extraction days) 

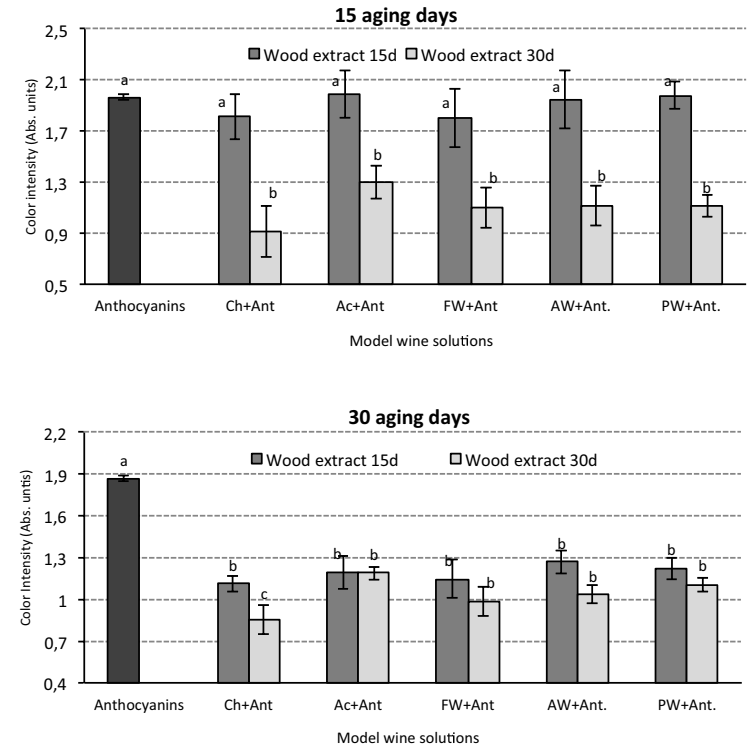

Figure 2. Color intensity quantified in model wine solutions containing different wood chip extracts after 15 and 30 aging days. $\mathrm{Ch}+\mathrm{Ant}=$ Cherry wood chip extract and anthocyanin extract; $\mathrm{Ac}+\mathrm{Ant}=$ Acacia wood chip extract and anthocyanin extract; $\mathrm{FW}+$ Ant $=$ French wood chip extract and anthocyanin extract; $\mathrm{AW}+\mathrm{Ant}=$ American wood chip extract and anthocyanin extract; PW + Ant $=$ Portuguese wood chip extract and anthocyanin extract.

and especially after 30 storage days. In wines, several works $[13,14]$ reported that the use of cherry barrels in wine aging induce a faster evolution of wine pigments with a quick augmentation formation of derived and polymeric compounds. In addition the use of this type of wood specie demonstrated to provide an environment favoring oxidative reactions and, thus increases the red color loss, making it less suitable for longer wine aging periods.

Concerning to CieLAB chromatic coordinates evaluation, Fig. 3 shows the $L^{*}, a^{*}$ and $C^{*}$ values obtained in model wine solutions after 30 aging days. For $L^{*}$ values (lightness), all model wine solutions (independently of the extraction time and wood species) showed higher lightness compared with the initial red model wine. This result is consistent with the decreased of color intensity showed in all red model wine after mixing with wood extracts. In fact, the decrease of color intensity induces a more lightness of the samples.

For $a^{*}$ values (redness), also a decrease of the red color component values was observed for all the mixtures of wood extracts with the red model wine. This result agrees the results already showed for color intensity. The decrease of red color component was similar for all model wine solutions, except for the model wine solutions containing cherry wood chip and French oak wood extract obtained after 30 extraction days, where the decrease was more evident.

Regarding to the results obtained for $c^{*}$ values (chroma), similar tendency was also detected. Thus, a tendency for a chroma values decrease was evident for all red model wine solutions mixed with wood chip extracts, corresponding also to a less color intensity.

Finally, the values obtained for color difference $(\Delta \mathrm{E})$ among red model wine solution alone and mixed with the different wood chip extracts (Fig. 4) showed that
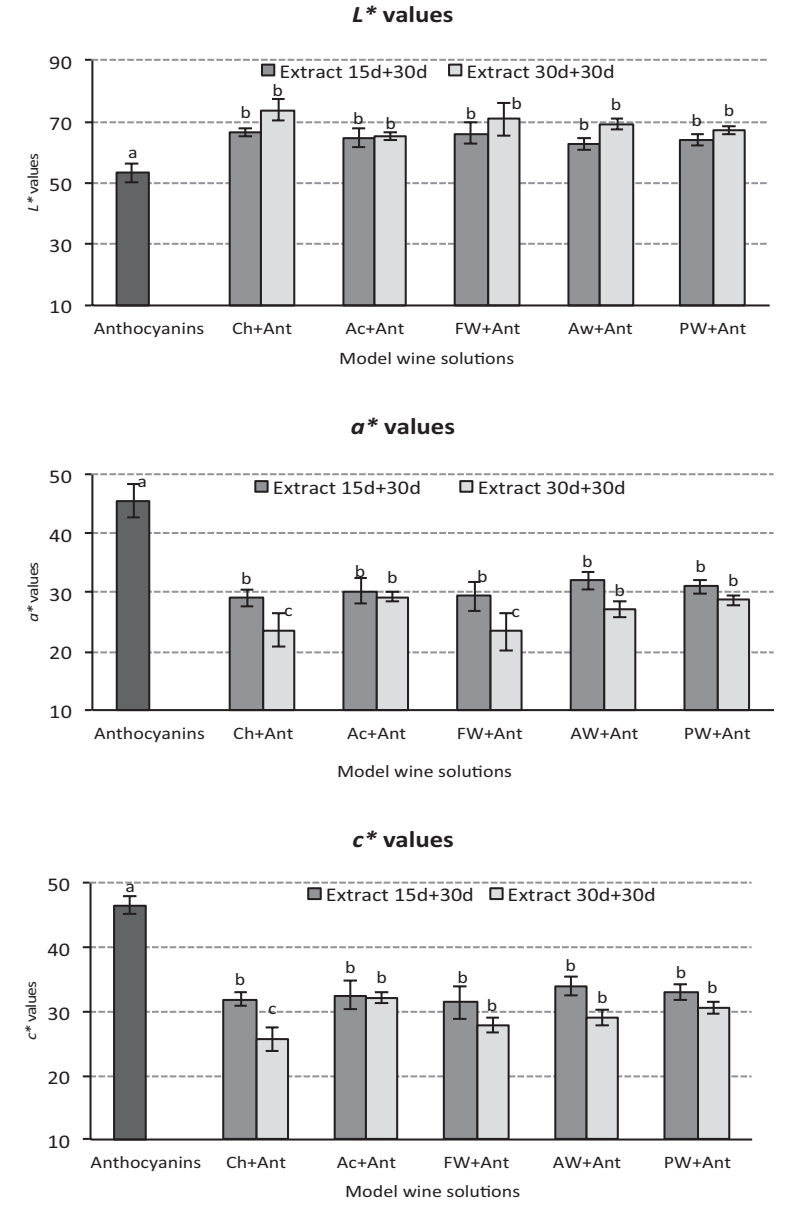

Figure 3. CieLab chromatic coordinates quantified in model wine solutions containing different wood chip extracts after 30 aging days. $\mathrm{Ch}+\mathrm{Ant}=$ Cherry wood chip extract and anthocyanin extract; Ac + Ant $=$ Acacia wood chip extract and anthocyanin extract; FW + Ant $=$ French wood chip extract and anthocyanin extract; AW + Ant $=$ American wood chip extract and anthocyanin extract; PW + Ant $=$ Portuguese wood chip extract and anthocyanin extract.

all mixture raised differences much higher than three CieLAB units. Thus, taking into account that $\triangle \mathrm{E}$ values of up to three CieLAB units indicates color differences appreciable to the human eyes [15], it could be affirmed that the contact between anthocyanin extract and wood chip extracts from different species led to color differences visually perceptible between in all model wine solutions. This result confirms the results already show for color intensity and the partial results obtained for CieLAB chromatic coordinates (Figs. 2 and 3). In addition, the high color differences results were independently of the wood chip extracts species. However, the model wine solutions containing wood extracts obtained with longer extraction time (30 days) showed significantly higher color difference values. Recently, Cejudo-Bastante et al. [16], conclude that the addition of oak wood chips (during both fermentation and aging) induced also a visually perceptible color differences from the early months of storage of a Syrah red wine (values among 5 and 12).

\subsection{Antioxidant capacity}

Figure 5 shows the antioxidant capacity quantified in the different model wine solutions after 30 aging days. 
$\Delta \mathrm{E}^{*}$ values

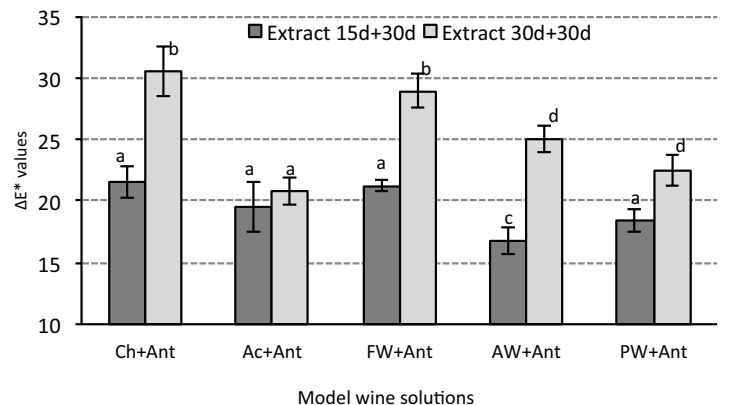

Figure 4. Color difference $(\Delta \mathrm{E})$ quantified in model wine solutions containing different wood chip extracts after 30 aging days. $\mathrm{Ch}+\mathrm{Ant}=$ Cherry wood chip extract and anthocyanin extract; Ac + Ant $=$ Acacia wood chip extract and anthocyanin extract; FW + Ant $=$ French wood chip extract and anthocyanin extract; AW + Ant $=$ American wood chip extract and anthocyanin extract; PW + Ant $=$ Portuguese wood chip extract and anthocyanin extract.
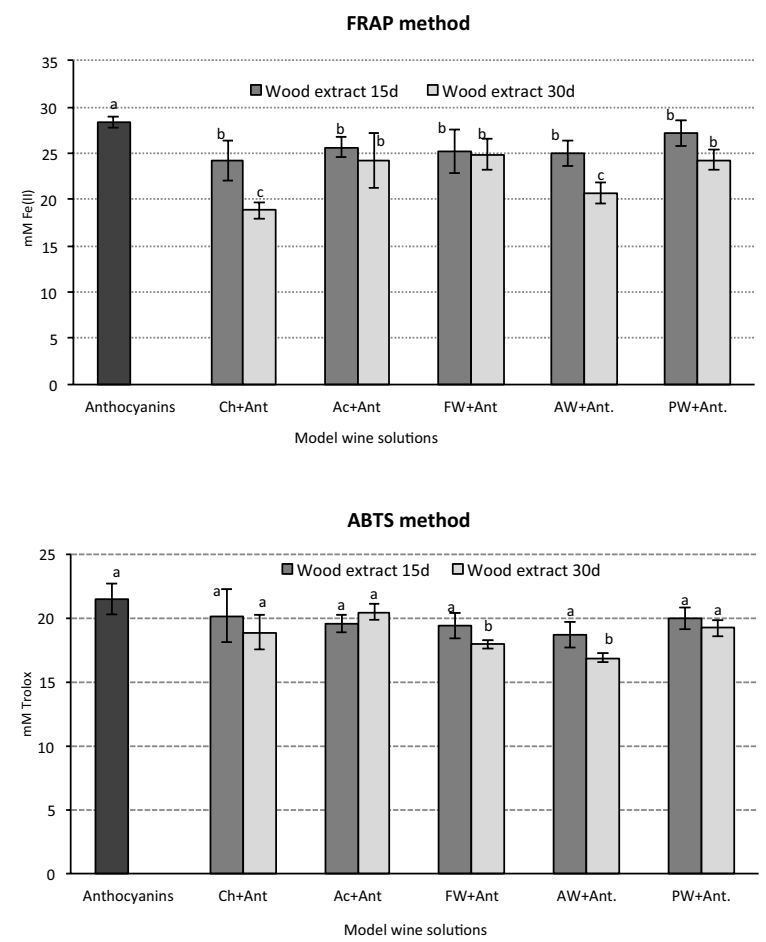

Figure 5. Antioxidant capacity quantified by two methodologies (FRAP and ABTS) in model wine solutions after 30 aging days. $\mathrm{Ch}+\mathrm{Ant}=$ Cherry wood chip extract and anthocyanin extract; Ac + Ant $=$ Acacia wood chip extract and anthocyanin extract; FW + Ant $=$ French wood chip extract and anthocyanin extract; AW + Ant $=$ American wood chip extract and anthocyanin extract; PW + Ant $=$ Portuguese wood chip extract and anthocyanin extract.

According to the results obtained, red model wine alone showed the highest antioxidant capacity (especially considering the results obtained by the use of FRAP method where the values were significantly higher) in comparison with the results obtained for model wine solutions containing also the different wood chip extracts. According to Rivero et al. [17], glucoside anthocyanins are one of the major anthocyanin group that determine the antioxidant capacity power of red wines, in particular malvidin-3-monoglucoside.

Concerning to the potential impact of the use of different wood chip extract species on antioxidant capacity, the results obtained were not totally distinctive. Thus, a slight tendency for lower antioxidant capacity values were obtained for the red model wine solutions mixed with cherry wood extract and with American oak wood extract (both extracted obtained after 30 extraction days). According to several authors $[4,18]$ the use of oak wood induce an increase of antioxidant capacity of the red wines during the aging process as a result of polyphenol compounds extraction. However, contrary results were obtained by Gortzi et al. [19]. For these authors, after a considerable aging period in contact with oak wood chips, red wines showed a decrease of polyphenolic content and consequently a reduction of antioxidant capacity. In addition, it is important to note that according to Psarra et al. [20] polyphenols extracted from oak wood chips yield almost 6.4 times higher reducing power comparative with acacia chips. Therefore, the antioxidant effects exerted by polyphenols do not depend exclusively on their total amount, but also on the different polyphenolic structures [21]. This fact could help to explain the similar values of antioxidant capacity found in the majority of the model wines solutions studied. Probably the use of a more extended aging period or higher wood chips concentration used to produce the wood chip extracts could be a factor to consider in the future to potentiate eventual differences.

\section{Conclusions}

In this work it was possible to detected a clear influence of the use of different wood chip species on evolution of the anthocyanin composition and consequently on chromatic parameters. This influence was especially detectable by a decrease of the color intensity and especially on red color component values. In addition, the antioxidant capacity showed also a slightly decrease of the values when anthocyanins were put in contact during 30 days with wood chip extracts. It was also clear that the time used to obtain the wood chip extracts had a particular influence on the results determining an increase of the variation of the parameters analyzed.

This research contributes to a better knowledge about the color evolution of red wines when they are aging in contact with oak wood and in especially when is using new alternative wood species, especially acacia and cherry. This aspect will be important if these wood species may come in the future to be authorized for oenological purposes.

António M. Jordão thanks to FCT (project UID/Multi/04016/ 2016) and IPV-CI\&DETS for their financial support. Authors also appreciated the collaboration of the companies that supplied the wood chip samples used in this study (SAI Enology, AEB Bioquímica and J.M. Gonçalves cooperage).

\section{References}

[1] I. Revilla, M.L. González-Sanjosé, M.C. GómezCordovés, Food Sci. Technol. Int. 5, 177-181 (1999)

[2] A.M. Jordão, J.M. Ricardo-da-Silva, O. Laureano, Am. J. Enol. Vitic. 57, 377 (2006) 
[3] A.M. Jordão, J.M. Ricardo-da-Silva, O. Laureano, W. Mullen, A. Cozier, Aust. J. Grape Wine Res. 14, 260 (2008)

[4] F.J. Gonçalves, A.M. Jordão, Ital. J. Food Sci. 21, 51 (2009)

[5] G. Coninck, A.M. Jordão, J.M. Ricardo-da-Silva, O. Laureano, J. Int. Sci. Vigne Vin 40, 25 (2006)

[6] M. Kyraleou, P.-L. Teissedre, E. Tzanakouli, Y. Kotseridis, N. Proxenia, K. Chira, I. Ligas, S. Kallithraka, Oeno One 50, 209 (2016)

[7] S. Pérez, M.L. González-Sanjosé, J. Agric. Food Chem. 52, 1181 (2004)

[8] Y. Glories, Connaiss. Vigne Vin 18, 253 (1984)

[9] S. Pérez, M.L. González-Sanjosé, Food Chem. 81, 301 (2003)

[10] M.D. Rivero, P. Muñiz, M.L. González-Sanjosé, J. Agric. Food Chem. 55, 5476 (2007)

[11] M. Sánchez, M.L. González-Sanjosé, S. Pérez, M. Ortega, C. González, J. Agric. Food Chem. 57, 11498 (2009)

[12] I. Revilla, M.L. González-Sanjosé, Eur. Food Res. Technol. 213, 281 (2001)
[13] F. Chinnici, N. Natali, F. Sonni, A. Bellachioma, C. Riponi, J. Agric. Food Chem. 59, 6575 (2011)

[14] M. De Rosso, A. Panighel, A. Dalla-Vedova, L. Stella, R. Flamini, J. Agric. Food Chem. 57, 1915 (2009)

[15] J.A. Martínez, M. Melgosa, M.M. Pérez, E. Hita, A.I. Negueruela, Food Sci. Technol. Int. 7, 439 (2001)

[16] M.J. Cejudo-Bastante, F.J. Rivero-Granados, F.J. Heredia, Eur. Food Res. Technol. 243, 575 (2017)

[17] M.D. Rivero, P. Muñiz, M.L. González-Sanjosé, Food and Chem. Toxicol. 46, 2815 (2008)

[18] A.M. Jordão, A.C. Correia, R. Del Campo, M.L. González-Sanjosé, Eur. Food Res. Technol. 235, 817 (2012)

[19] O. Gortzi, X. Metaxa, G. Mantanis, S. Lalas, Food Chem. 141, 2887 (2013)

[20] C. Psarra, O. Gortzi, D.P. Makris, J. Inst. Brew. 121, 207 (2015)

[21] M. Aoun, D.P. Makris, Inter. J. Food Studies 2, 238 (2013) 